\title{
Study of Prostatic Fluid from Patients with Elevated Levels of Prostate-Specific Antigen
}

\author{
Haiwen Chen ${ }^{1}$, Narumi Tsuboi ${ }^{2}$, Taiji Nishimura ${ }^{2}$, Yoshihiko Norose ${ }^{3}$, Yuka Saito ${ }^{2}$, \\ Ryoji Kimata ${ }^{2}$, Yukihiro Kondo ${ }^{2}$ and Go Kimura ${ }^{2}$ \\ ${ }^{1}$ Department of Urology, Second Hospital, Xi'an Jiaotong University, China \\ ${ }^{2}$ Department of Urology, Nippon Medical School \\ ${ }^{3}$ Department of Microbiology and Immunology, Nippon Medical School
}

\begin{abstract}
Introduction: Characteristics of prostatic fluid $(\mathrm{PF})$, which can be obtained in large amounts during screening transrectal ultrasound just before prostate biopsy to detect prostate cancer, were investigated. These characteristics include the amount of PF obtained and the number of leukocytes in PF, which would be useful for planning cell-biological or immunological studies of leukocytes in PF and for increasing the understanding of prostatitis in elderly men.

Patients and Methods: The volume of PF and the number of leukocytes in PF were measured in 50 patients suspected of having prostate cancer because of elevated levels of serum prostate-specific antigen (PSA). Correlations of the volume of $\mathrm{PF}$, the number of leukocytes per milliliter, the total leukocyte number with age and prostate volume and correlation of PSA levels with the number of leukocytes per milliliter and total leukocyte number were also investigated.
\end{abstract}

Results: The average patient age was 67.2 years, and PF specimens were obtained from 43 of the 50 patients (86\%). The mean \pm SD of PF volume, number of leukocytes in PF, and total leukocyte number were $347.65 \pm 305.76 \mu l, 4.84 \pm 6.07 \times 10^{6} / \mathrm{ml}$, and $1.47 \pm 2.10 \times 10^{6}$, respectively. A correlation was observed only between the total leukocyte number and the volume of the transitional zone $(\mathrm{P}=0.039)$.

Conclusions: These data provide information for investigators to plan cell-biological or immunological studies of leukocytes in PF and for understanding prostatitis in elderly men.

(J Nippon Med Sch 2006; 73: 24-28)

Key words: prostatic fluid, leukocyte subpopulation, prostate, prostate-specific antigen

\section{Introduction}

Many papers over the past 50 years have described bacterial cultures and leukocyte counts of prostatic fluid $(\mathrm{PF})$ from healthy subjects and patients with prostatitis to clarify the significance of leukocytosis in $\mathrm{PF}^{1.2}$. However, at the same time,

Correspondence to Haiwen Chen, Department of Urology, Second Hospital, Xi'an Jiaotong University, No. 157 Xi Wu Road, Xi'an 710004, China

E-mail: xawenzi168@yahoo.com

Journal Website (http://www.nms.ac.jp/jnms/) 
investigators have had difficulty obtaining $\mathrm{PF}$, as $\mathrm{Nickel}^{3}$ described "expressed prostatic secretions cannot be obtained at the meatus urethrae following prostatic massage in a considerable percentage of cases." Therefore, urine obtained after prostate massage has been used instead of PF. Consequently, few cell-biological or immunological studies of leukocytes in PF have been performed ${ }^{4-10}$.

However, we recently found that PF is easily obtained at screening transrectal ultrasound (TRUS) of the prostate just before biopsy in most patients because TRUS allows more effective prostate massage than does the finger of the examiner. A literature search yielded no earlier paper describing the volume of PF and the number of leukocytes in PF obtained just before prostate biopsy from patients suspected of having prostate cancer. We believe such a study would be valuable because it would provide basic information for planning cellbiological or immunological studies of leukocytes in $\mathrm{PF}$ and for increasing the understanding of prostatitis in elderly men, even when specimens are obtained from patients suspected of having prostate cancer rather than patients with prostatitis.

\section{Patients and Methods}

Form April 2005 through June 2005, 50 men underwent prostate biopsy under general anesthesia in our institution because of elevated serum levels of prostate-specific antigen except in patients 16 and 20 (Table 1). Patient 16 consulted us because of abnormal blood flow in the right lobe detected with power Doppler ultrasonography performed by a local doctor, and patient 20 consulted us because of a suspected neuroendocrine tumor of the prostatic urethra. None of the patients presented with symptoms of prostatitis such as urethral burning, increase urinary frequency, and gross hematuria. Patient ages ranged from 52 to 84 years, with an average age of 67.2 years.

After general anesthesia, the patient was placed in the lithotomy position. First, TRUS was performed. The ultrasound probe touched the rectal wall behind prostate tightly, and examination was thoroughly performed. The probe was moved from proximal to distal, and from left to right. Digital massage was then performed as usual to collect PF.

PF was collected in a tube containing $100 \mu l$ of phosphate-buffered solution (PBS) during screening TRUS just before transrectal biopsy of the prostate. The total prostate volume and the transitional zone (TZ) volume under TRUS were also recorded.

The volume of PF was calculated by substracting the volume of PBS from the whole volume in the tube after collection of PF.

The leukocyte count was determined with a hemocytometer, and the leukocyte number per milliliter and the total leukocyte number obtained in each case were recorded.

Correlations of PF volume, leukocyte number per milliliter and total leukocyte number with age, total prostate volume, and TZ volume and of PSA levels with the number of leukocytes per milliliter and the total leukocyte number were also investigated.

Statistical analysis was performed with Student's $t$-test.

Informed consent for obtaining $\mathrm{PF}$ and for the use of PF and biopsy specimens for possible investigation were obtained from each patient before biopsy.

\section{Results}

Background characteristics of all patients and the results are shown in Table $\mathbf{1}$. PF was obtained from 43 of 50 patients $(86 \%)$. The volume of $\mathrm{PF}$ ranged from 20 to $1,140 \mu l$, with a mean \pm SD of $347.65 \pm$ $305.76 \mu l$. The leukocyte number per milliliter ranged from $3.8 \times 10^{4}$ to $2.8 \times 10^{7} / \mathrm{m} l$ with a mean \pm $\mathrm{SD}$ of $4.84 \pm 6.07 \times 10^{6} / \mathrm{ml}$. The total leukocyte number ranged from $4.4 \times 10^{3}$ to $8.2 \times 10^{6}$ with a mean \pm SD of $1.47 \pm 2.10 \times 10^{6}$.

In 43 patients from whom PF was obtained, there was no correlation between PF volume and age $(\mathrm{p}=$ $0.419)$, total volume of the prostate $(p=0.234)$, or $\mathrm{TZ}$ volume $(p=0.217)$, or between leukocyte number per milliliter and age $(p=0.884)$, total volume of the prostate $(\mathrm{p}=0.338)$, or $\mathrm{TZ}$ volume $(\mathrm{p}=0.188)$. Furthermore, there was no correlation between total leukocyte number and age $(p=0.422)$ or the total volume of the prostate $(p=0.155)$. However, there 
H. Chen, et al

Table 1 Patient characteristics

\begin{tabular}{|c|c|c|c|c|c|c|c|}
\hline No & Age & TPSA & TPV & $\mathrm{TZV}$ & $\mathrm{PF}(\mu l)$ & $\begin{array}{c}\text { Cell } \\
\text { Count }(\mathrm{m} l)\end{array}$ & Total Cells \\
\hline $1^{*}$ & 68 & 10.0 & 20.2 & 6.7 & 0 & 0 & 0 \\
\hline 2 & 62 & 8.7 & 34.5 & 17.1 & 100 & $2.00 \times 10^{7}$ & $2.00 \times 10^{6}$ \\
\hline 3 & 57 & 4.3 & 29.2 & 11.9 & 50 & $4.00 \times 10^{6}$ & $8.00 \times 10^{4}$ \\
\hline 4 & 75 & 9.2 & 27.8 & 9.4 & 110 & $3.40 \times 10^{6}$ & $3.70 \times 10^{5}$ \\
\hline 5 & 74 & 4.2 & 28.8 & 9.3 & 170 & $1.10 \times 10^{7}$ & $1.80 \times 10^{6}$ \\
\hline 6 & 52 & 17.0 & 32.7 & 16.4 & 0 & 0 & 0 \\
\hline $7^{*}$ & 65 & 6.1 & 23.1 & 6.5 & 58 & $5.27 \times 10^{6}$ & $3.00 \times 10^{5}$ \\
\hline 8 & 58 & 7.8 & 27.8 & 6.9 & 600 & $1.13 \times 10^{6}$ & $6.84 \times 10^{5}$ \\
\hline $9^{*}$ & 73 & 12.0 & 42.3 & 25.5 & 31 & $1.30 \times 10^{7}$ & $4.00 \times 10^{5}$ \\
\hline $10^{*}$ & 67 & 64.0 & 19.5 & 5.0 & 301 & $1.55 \times 10^{6}$ & $4.68 \times 10^{5}$ \\
\hline $11^{*}$ & 69 & 69.0 & 29.4 & 10.0 & 0 & 0 & 0 \\
\hline $12^{*}$ & 65 & 12.0 & 16.8 & 3.5 & 55 & $1.05 \times 10^{7}$ & $5.83 \times 10^{5}$ \\
\hline 13 & 61 & 10.0 & 56.0 & 36.9 & 400 & $2.04 \times 10^{7}$ & $8.15 \times 10^{6}$ \\
\hline $14^{*}$ & 61 & 36.0 & 28.0 & 4.6 & 815 & $5.24 \times 10^{6}$ & $3.91 \times 10^{6}$ \\
\hline 15 & 76 & 17.0 & 73.4 & 46.9 & 162 & $2.80 \times 10^{7}$ & $4.50 \times 10^{6}$ \\
\hline 16 & 57 & 2.9 & 39.0 & 16.9 & 440 & $1.40 \times 10^{6}$ & $6.10 \times 10^{5}$ \\
\hline $17^{*}$ & 60 & 5.6 & 36.1 & 25.6 & 682 & $1.20 \times 10^{7}$ & $8.20 \times 10^{6}$ \\
\hline $18^{*}$ & 66 & 19.0 & 37.8 & 12.0 & 188 & $1.44 \times 10^{6}$ & $2.70 \times 10^{5}$ \\
\hline 19 & 75 & 6.4 & 74.9 & 53.3 & 151 & $1.76 \times 10^{6}$ & $2.70 \times 10^{5}$ \\
\hline $20^{*}$ & 62 & 3.1 & 51.2 & 30.4 & 120 & $1.00 \times 10^{6}$ & $1.20 \times 10^{5}$ \\
\hline 21 & 64 & 7.5 & 20.0 & 2.7 & 20 & $2.20 \times 10^{6}$ & $4.40 \times 10^{3}$ \\
\hline 22 & 76 & 4.2 & 57.7 & 41.8 & 1,140 & $3.84 \times 10^{4}$ & $4.37 \times 10^{6}$ \\
\hline 23 & 62 & 5.5 & 32.1 & 11.1 & 150 & $1.48 \times 10^{6}$ & $2.20 \times 10^{5}$ \\
\hline 24 & 59 & 9.5 & 55.3 & 39.9 & 451 & $1.02 \times 10^{7}$ & $4.60 \times 10^{6}$ \\
\hline 25 & 59 & 16.0 & 26.9 & 12.5 & 160 & $3.05 \times 10^{5}$ & $4.90 \times 10^{4}$ \\
\hline 26 & 69 & 14.0 & 56.6 & 38.9 & 865 & $6.40 \times 10^{6}$ & $5.50 \times 10^{6}$ \\
\hline $27^{*}$ & 62 & 4.3 & 35.6 & 9.1 & 212 & $2.04 \times 10^{6}$ & $4.30 \times 10^{5}$ \\
\hline 28 & 73 & 14.0 & 58.1 & 39.1 & 141 & $7.04 \times 10^{6}$ & $9.90 \times 10^{5}$ \\
\hline $29^{*}$ & 71 & 15.0 & 16.1 & 4.3 & 0 & 0 & 0 \\
\hline 30 & 70 & 10.0 & 53.2 & 29.5 & 283 & $2.16 \times 10^{6}$ & $6.10 \times 10^{5}$ \\
\hline $31^{*}$ & 64 & 18.0 & 19.5 & 8.8 & 140 & $4.00 \times 10^{6}$ & $5.60 \times 10^{5}$ \\
\hline 32 & 62 & 7.7 & 35.9 & 19.1 & 860 & $5.24 \times 10^{6}$ & $4.50 \times 10^{6}$ \\
\hline $33^{*}$ & 74 & 42.0 & 17.1 & 3.7 & 0 & 0 & 0 \\
\hline $34^{*}$ & 80 & 9.0 & 43.5 & 30.8 & 0 & 0 & 0 \\
\hline $35^{*}$ & 80 & 18.0 & 20.7 & 8.9 & 172 & $2.16 \times 10^{6}$ & $3.72 \times 10^{5}$ \\
\hline $36^{*}$ & 67 & 7.3 & 31.5 & 15.0 & 170 & $5.12 \times 10^{6}$ & $8.70 \times 10^{5}$ \\
\hline 37 & 68 & 7.0 & 32.9 & 18.4 & 622 & $2.36 \times 10^{6}$ & $1.47 \times 10^{6}$ \\
\hline 38 & 61 & 8.8 & 42.1 & 18.6 & 663 & $4.90 \times 10^{5}$ & $3.25 \times 10^{5}$ \\
\hline $39^{*}$ & 65 & 4.3 & 26.5 & 5.6 & 1,012 & $1.38 \times 10^{6}$ & $1.40 \times 10^{6}$ \\
\hline 40 & 68 & 4.4 & 96.5 & 59.3 & 310 & $5.00 \times 10^{5}$ & $1.55 \times 10^{5}$ \\
\hline $41^{*}$ & 66 & 73.6 & 22.4 & 8.4 & 62 & $3.12 \times 10^{6}$ & $1.93 \times 10^{5}$ \\
\hline 42 & 78 & 4.4 & 42.6 & 27.4 & 0 & 0 & 0 \\
\hline 43 & 62 & 4.6 & 35.4 & 17.9 & 940 & $1.28 \times 10^{6}$ & $1.20 \times 10^{6}$ \\
\hline $44^{*}$ & 56 & 66.0 & 38.3 & 18.6 & 228 & $9.20 \times 10^{5}$ & $2.10 \times 10^{5}$ \\
\hline $45^{*}$ & 76 & 5.4 & 51.8 & 30.4 & 263 & $1.84 \times 10^{6}$ & $4.84 \times 10^{5}$ \\
\hline 46 & 65 & 6.9 & 106.5 & 68.7 & 642 & $1.10 \times 10^{6}$ & $7.06 \times 10^{5}$ \\
\hline $47^{*}$ & 80 & 13.0 & 17.8 & 5.1 & 558 & $5.8 \times 10^{5}$ & $3.24 \times 10^{5}$ \\
\hline $48^{*}$ & 84 & 10.0 & 21.8 & 10.0 & 174 & $1.40 \times 10^{6}$ & $2.44 \times 10^{5}$ \\
\hline $49^{*}$ & 72 & 20.0 & 20.6 & 9.1 & 38 & $1.20 \times 10^{6}$ & $4.56 \times 10^{4}$ \\
\hline 50 & 62 & 8.1 & 39.1 & 18.0 & 240 & $2.54 \times 10^{6}$ & $6.10 \times 10^{5}$ \\
\hline
\end{tabular}

* Italicized numbers indicate the patient was diagnosed with cancer.

TPSA: total PSA, TPV: total prostate volume, TZV: TZ volume, PF: volume of PF. 
was a correlation between the total leukocyte number and the $\mathrm{T} Z$ volume $(\mathrm{p}=0.039)$. There was no correlation between PSA level and leukocyte number per milliliter $(\mathrm{p}=0.675)$ or total leukocyte number $(\mathrm{p}=0.503)$.

There was no significant difference between 43 patients from whom PF was obtained and 7 patients from whom PF was not obtained concerning age $(\mathrm{p}=$ $0.228)$, total prostate volume $(\mathrm{p}=0.178)$, or $\mathrm{TZ}$ volume $(\mathrm{p}=0.320)$.

Cancer was present in 24 of 50 patients (48\%). In 19 patients with cancer and 24 patients without cancer the average leukocyte number per milliliter was $3.88 \times 10^{6} / \mathrm{ml}$ and $1.02 \times 10^{6} / \mathrm{ml}$, respectively, and the average total leukocyte number was $5.60 \times$ $10^{6}$ and $1.82 \times 10^{6}$, respectively. There was no significant difference between patients with cancer and patients without cancer in the leukocyte number per milliliter $(p=0.363)$ or the total leukocyte number $(\mathrm{p}=0.218)$.

\section{Discussion}

Recently, there have been an increasing number of papers regarding the relationship between prostate cancer and chronic inflammation ${ }^{11}$ which has not been confirmed. There have also been an increasing number of studies examining the role of dendritic cells in the prostate tissue regardless of inflammation or cancer ${ }^{12}$. However, few studies have examined leukocytes in PF, because it is very difficult to obtain large numbers of leukocytes in PF. Recently we found that large amounts of PF and leukocytes in PF could be obtained by massage with TRUS of the prostate before prostate biopsy. Using this method in the present study, we provide information concerning available $\mathrm{PF}$ volume and leukocytes in PF before planning these experiments. For example, in our previous study of bcl-2 expression by leukocytes in PF from patients with prostatitis, the minimal number of total leukocytes required for the experiments in each case was $1.0 \times$ $10^{6}{ }^{10}$. In the present study, the average total leukocyte number obtained in each case was $1.47 \times$ $10^{6}$; however, more than $1.0 \times 10^{6}$ leukocytes were obtained in only $26.0 \%$ of patients (13 of 50 patients)
(Table 1). Therefore, for example, if a researcher attempts an experiment using $1.0 \times 10^{6}$ leukocytes in PF from 50 patients, obtaining specimens from 193 (50/0.26) patients would be necessary to complete the experiment.

We examined the correlations PF volume, leukocyte number per milliliter, and total leukocyte number with age, total prostate volume, and the volume of the $\mathrm{TZ}$, where most prostate glands and ducts exist. We found a correlation only between total leukocyte number and $\mathrm{TZ}$ volume $(\mathrm{P}=0.039)$. More marked inflammation in a larger area of hyperplasia in the $\mathrm{TZ}$ may be the reason for this difference. However, this issue remains to be studied.

The present findings may also be useful for increasing the understanding of prostatitis in elderly men. It is believed that benign prostatic hypertrophy accompanies inflammation ${ }^{13}$. We reconfirmed this by finding an average leukocyte number of $4.8 \times 10^{6}$ per milliliter in PF from elderly patients, although none of the patients had symptoms of urinary tract infection.

Using the results of leukocytosis in patients who underwent prostate biopsy in the present study, we are now undertaking a correlative study of leukocytosis in PF and in prostate tissue from prostatectomy specimens in the same patients, and of subpopulations of leukocytes in PF and prostate tissue.

In conclusion, a larger amount of $\mathrm{PF}$ and a larger number of leukocytes in PF were obtained by massage during screening TRUS of the prostate before prostate biopsy for the detection of prostate cancer. That is, massage by this method was superior to digital massage, and this method allows cell-biological and immunological experiments of leukocytes in $\mathrm{PF}$. The present data, such as the average volume of PF and leukocytes in PF will provide information for planning the aforementioned experiments of leukocytes in PF. The present results may also be useful for increasing the understanding of prostatitis in elderly men because leukocytosis in PF with an average of $4.8 \times 10^{6}$ leukocytes per milliliter was observed, even though patients had no symptoms of urinary tract infection. 


\section{References}

1. Ghormley KO, Cook ED, Needham GM: Bacterial flora in chronic prostatitis. Amer J Clin Path 1954; 24: $186-193$.

2. O'Shaugnessy EJ, Parrino PS, White JD: Chronic prostatitis-fact or fiction. JAMA 1956; 160: 540-542.

3. Nickel JC: The pre- and post-massage test (PPMT): A simple screen for prostatitis. Tech Urol 1997; 3: 38-43.

4. Anderson RU, Weller C: Prostatic secretion leukocyte studies in non-bacterial prostatitis (prostatosis). J Urol 1979; 121: 292-294.

5. Nishimura T, Kanamori S, Akimoto M, Kawai H: Macrophages in prostatic fluid. Br J Urol 1980; 52: 381-385.

6. Nishimura T, Kanamori S, Yoshida K, Akimoto M, Kawai H: Longitudinal study of macrophages in prostatic fluid from nonbacterial prostatitis patients. Urology 1981; 18: 255-257.

7. Nishimura T, Terashima Y, Hattori T, Satoh M, Yoshida K, Akimoto M: Study of macrohpages in prostatic fluid from nonbacterial prostatitis patients. V. Relation between activation of macrophages and stage of prostatitis. Uro Int 1991; 46: 15-17.

8. Nishimura $T$, Abe $H$, Ito $H$, Ikeda $K$, Oka $F$, Yamamoto M: IL-1ra versus IL-1 levels in prostatic fluid from prostatitis patients. Uro Int 1998; 60: 9296.

9. Ludwig M, Steltz C, Huwe P, Schaffer R, Altmannsberger $\mathrm{M}$, Weidner W: Immunocytological analysis of leukocyte subpopulations in urine specimens before and after prostatic massage. Eur Urol 2001; 39: 277-282.

10. Abe H, Nishimura T, Asoh S, Ohta S: Expression of Bcl-2 on leukocytes in prostatic fluid from patients with acute and chronic prostatitis. J Infect Chemother 2003; 9: 156-159.

11. Roberts RO, Bergstralh EJ, Bass SE, Lieber MM, Jacobsen SJ: Prostatitis as a risk factor for prostate cancer. Epidemiology 2004; 15: 93-99.

12. Tourkova IL, Yamabe K, Foster B, Chatta G, Perez L, Shurin GV, Shurin MR: Murine prostate cancer inhibits both in vivo and in vitro generation of dendritic cells from bone marrow precurcors. Prostate 2004; 59: 203-213.

13. Perez-Blas M, Martinez-Martin B, Carballido J, Hontoria J, Salazar LI, Olivier C, Alvarez-Mon M: Defective functional response to membrane stimuli in lymphocytes from patients with benign prostatic hyperplasia. Clin Exp Immunol 1995; 101: 521-526.

(Received, October 4, 2005)

(Accepted, January 6, 2006) 